\title{
QUALIDADE DE VIDA DE IDOSOS AMAZÔNICOS QUE PARTICIPAM DE UM GRUPO DE CONVIVÊNCIA
}

Marcela Alves de Oliveira ${ }^{1}$ Hemellen Ferreira Ribeiro', Nadia Pinheiro da Costa ${ }^{2}$ https://orcid.org/0000-0003-2987-7861
https://orcid.org/0000-0002-5764-1672
https://orcid.org/0000-0001-6550-366X

Objetivo: avaliar a qualidade de vida de idosos participantes de um grupo de convivência e verificar o perfil sociodemográfico. Metodologia: Trata-se de um estudo com abordagem quantitativa do tipo descritiva, onde foi aplicado um questionário sociodemográfico e o ins-trumento Whoqol-Bref para avaliar a qualidade de vida de 103 idosos de um centro de convi-vência de Belém do Pará. Resultados: a maioria são idosas pensionistas/aposentadas na faixa etária entre 70-75 anos, com escolaridade de 8 anos ou mais. Em relação ao instrumento Who-qol-bref os idosos apresentaram maior escore no domínio de percepção da qualidade de vida e nas relações sociais seguidos respectivamente pelos físico, psicológico, satisfação com a saúde e por último meio ambiente. Conclusão: A maioria das mulheres idosas aposentadas demons-traram possuir boa qualidade de vida, destacado para o domínio desta percepção e das rela-ções sociais.

Descritores: Idosos; Qualidade de vida; Enfermagem.

\section{QUALITY OF LIFE OF AMAZONIAN ELDERLY WHO PARTICIPATE IN A CO-EXISTENCE GROUP}

Objective: to evaluate the quality of life of elderly participants of a coexistence group; verify the sociodemographic profile. Methodology: This is a descriptive quantitative study, where a sociodemographic questionnaire and the Whoqol-Bref instrument were used to evaluate the quality of life of 103 elderly people from a community center in Belém do Pará. Results: most of them are elderly with age range between 70-75 years, schooling 8 years or more, pen-sioned/retired. In relation to the Whoqol-bref instrument, the elderly presented a higher score in the domain of perception of quality of life and social relations, followed respectively by physical, psychological, health satisfaction and ambient environment. Conclusion: Most retired elderly women demonstrated to have a good quality of life, highlighted for the domain of this perception and social relations.

Descriptors: Elderly; Quality of life; Nursing.

\section{CALIDAD DE VIDA DE ANCIANOS AMAZÓNICOS QUE PARTICIPAN EN UN GRUPO DE CONVIVENCIA}

Objetivo: evaluar la calidad de vida de los ancianos participantes de un grupo de convivencia; verificar el perfil sociodemográfico. Metodología: Se trata de un estudio con abordaje cuanti-tativo del tipo descriptivo, donde se aplicó un cuestionario sociodemográfico y el instrumento Whoqol-Bref para evaluar la calidad de vida de 103 ancianos de un centro de convivencia de Belém do Pará. Resultados: la mayoría son mujeres, tienen rango de edad entre 70-75 años, escolaridad 8 años o más, pensionista/jubilado. En cuanto al instrumento Whoqol-bref, los ancianos presentaron mayor puntuación en el dominio de percepción de la calidad de vida y relaciones sociales seguido respectivamente por la satisfacción física, psicológica, de salud y, por último, el medio ambiente. Conclusión: La mayoría de las mujeres ancionas fueran jubila-das demostraron tener una buena calidad de vida, destacada por el dominio de esta percepción y las relaciones sociales..

Descriptores: Ancianos; Calidad de vida; Enfermería. 


\section{INTRODUÇÂO}

A população brasileira vem passando por uma transformação demográfica, e com isso a expectativa de vida dos idosos está aumentando. A Organização Mundial de Saúde (OMS) considera idoso um limite de 65 anos ou mais de idade para os indivíduos de países desenvolvidos e 60 anos ou mais de idade para individuos de países subdesenvolvidos"(1).

No Brasil é notório o aumento da população de pessoas com 60 anos ou mais, devido à diminuição da mortalidade e da natalidade. Estima-se que em 2025 o Brasil poderá se tornar o sexto país com maior número de população de idosos em números absolutos, com cerca de 34 bilhões de idosos ${ }^{(2)}$.

Sabe-se que o envelhecimento não ocorre de forma semelhante a todos os individuos; os idosos podem envelhecer de forma saudável (senescência), mas alguns estão sujeitos a patologia, alterações físicas, cognitivas e sociais (senilidade) necessitando de profissionais capacitados para atenderem de forma adequada e humanizada essa população, proporcionando não só um tempo de vida maior, mas também qualidade de vida ${ }^{(3)}$.

Nesse contexto busca-se envelhecer com qualidade de vida e esta pode ser alcançada por meio da promoção do acesso à saúde, através da vacinação, alimentação saudável e de qualidade, atividade física, lazer, condições de moradia, as relações de convivência. "A qualidade de vida é um conceito amplo e subjetivo, atrelado ao bem-estar sociocultural, físico e psicológico dos indivíduos, sendo sinônimo de saúde e longevidade"(4).

No Brasil o crescimento da população de idosos ocorre de forma acelerada e a qualidade de vida precisa acompanhar esse crescimento. ,Vvisto que muitos idosos permanecem em situações desfavoráveis, uma estratégia importante para estes é a implementação de grupos de convivência na comunidade, sendo um fator relevante como meio de prevenção de agravos na velhice, pois a participação em grupos promove a interação interpessoal, autoestima, autonomia, independência, tornando-se mais ativos e saudáveis ${ }^{(5)}$.

Dessa forma, nessa pesquisa foi avaliar a qualidade de vida dos idosos que frequentam regularmente um grupo de convivência em Belém do Pará.

\section{METODOLOGIA}

\section{Tipo de estudo}

Estudo descritivo, de abordagem quantitativa.

\section{Participantes da pesquisa:}

O público alvo dessa pesquisa foram idosos a partir de 60 anos de idade. A casuística estudada foi composta por uma amostra de idosos a partir de 60 anos que frequentam regularmente as atividades desenvolvidas no Centro da Terceira Idade palácio Bolonha. Considerando que a população de estudo se constitui de 1.070 idosos, calculou-se o valor do $n$ (amostral) a ser utilizada nesta pesquisa, conforme a fórmula citada por Fontelles ${ }^{(6)}$ demonstrada abaixo:

$$
n=\frac{Z^{2} \times P \times Q \times N}{e^{2} \times(N-1)+Z^{2} \times P \times Q}
$$

\section{Sendo:}

Z = Nível de Confiança (95\%)

$\mathrm{P}=$ Quantidade de acerto esperado (95\%)

$\mathrm{Q}=$ Quantidade de Erro esperado (5\%)

$\mathrm{N}=$ População Total $=103$ idosos

e = Nivel de Precisão (5\%)

O cálculo foi feito admitindo um nível de confiança de 95\% e um erro amostral de $5 \%$. Após o cálculo, o (n) amostral total foi composto por 103 idosos. O método para seleção dos participantes ocorreu por amostragem aleatória simples. A amostra atingiu mais de $70 \%$ da população de idosos.

\section{Critérios de inclusão}

Idosos com idade igual ou superior a 60 anos que frequentam regularmente as atividades desenvolvidas no Centro de Convivência Palácio Bolonha.

\section{Critérios de exclusão}

Foram excluidos do estudo idosos que não tinham atendimento regular ou que possuíam déficit cognitivo, dessa forma antes de começarmos a pesquisa foi realizada uma avaliação da cognição desses idosos, através do instrumento Mini Exame do Estado Mental (MEEM) que é constituído de duas secções, a primeira diz respeito a respostas vocais, orientação, memória e atenção; e a segunda compreende a linguagem (capacidade para nomear, obedecer a comandos, escrita e desenho) que tem como pontuação máxima 30 pontos. Adotou-se como nota de corte, 23 pontos para alfabetizados e 19 para analfabetos ${ }^{(7)}$.

\section{Local do estudo}

O presente estudo foi realizado com idosos participantes do grupo de convivência do Centro da Terceira Idade Palácio Bolonha que está localizado no estado de Belém- PA. O Centro é uma organização não governamental com cerca 1.170 cadastrados e tem como público alvo pessoas a partir dos 40 anos de idade.

O Centro dispõe de profissionais fisioterapeutas, terapeuta ocupacional e educador físico, não contando 
com o profissional de enfermagem. Oferece atividades tais como: alongamento; confecção de artesanato; boxe; coral; dança de salão; dança do ventre; aula de francês; ginástica localizada, hidroginástica, aulas de informática, musculação; memorização; natação; pilates, solo e studio; pintura em tela e tecido; ritmos, aulas sobre manuseio de smartphone, yoga; viagens e festas.

\section{Coleta dos dados}

A coleta de dados foi realizada no 2 ㅇ semestre de 2017 , no Centro da Terceira Idade Palácio Bolonha, no Município de Belém, Estado do Pará, após aprovação do Comitê de Ética em Pesquisa da Fundação Santa Casa de Misericórdia do Pará ( $n^{\circ}$ do parecer CAAE 78435917.5.0000.5171.). Todos os idosos participantes desta pesquisa assinaram o Termo de consentimento Livre e Esclarecido (TCLE)

Os instrumentos foram aplicados com cada idoso da seguinte forma: Primeiramente foi avaliado a cognição através do Mini Exame do Estado Mental, posteriormente foram coletados os dados do perfil sociodemográfico (sexo, idade, escolaridade, sabe ler,cor/raça, situação conjugal, possui alguma doença, etc) por meio de um instrumento que elaboramos com base na caderneta de saúde da pessoa idosa.

Por último avaliamos a qualidade de vida dos participantes através do instrumento WHOQOL-BREF, que é um questionário constituido de 26 perguntas sobre qualidade de vida, no qual as duas primeiras avaliam a percepção e a satisfação que os idosos têm com a qualidade de vida, e o restante é distribuído em quatro domínios: Físico; Psicológico; Relações sociais e Meio ambiente ${ }^{(8)}$.

\section{Procedimentos de análise dos dados}

Neste estudo utilizamos o teste não paramétrico Quiquadrado de Pearson para tendência/aderência e correlação entre as variáveis nominais, simbolizado por $\chi^{2}$, que é um teste de hipóteses que se destina a verificar se há tendência / correlação significativa ou não na ocorrência de determinados fatos, mensurados de forma nominal, adotando-se um nível de significância de $\mathrm{p}$-valor < 0,05 ou p-valor < 0,10(9).

Desta forma, os dados coletados foram tabulados, interpretados, processados e analisados por meio da estatística descritiva e inferencial. Para a análise dos dados foram utilizados recursos de computação, por meio do processamento no sistema Microsoft Excel e Statistic Package for Social Sciences (SPSS) versão 24.0, todos em ambiente Windows 7.

\section{RESULTADOS}

A Tabela 1 mostra o perfil sociodemográfico dos idosos participantes da pesquisa. Nela, verifica-se que a maioria significativa $(p<0.05)$ dos idosos pesquisados são do sexo feminino (93; 90,29\%) e com faixa etária predominante de 70 a 75 anos (32; 31,07\%). Entre os idosos predomina a cor e/ou raça parda (48; 46,60\%), todos declararam saber ler e escrever (103=100\%). Nota-se que a maioria dos idosos possuem 8 anos ou mais de escolaridade (78=75,73\%), é aposentado ou pensionista $(89=86,41 \%)$ e recebe três ou mais salários mínimos (55=53,40\%). A maioria dos idosos reside com parentes $(65=63,11 \%)$.

No que refere a situação conjugal, observa-se que a maioria é viúva (o) (33=32,04\%). Os que são casados (as) ou que convivem com parceiro (a) também se destacam (30= $29,13 \%)$

A maioria declarou que não possui deficiência $(77=74,76 \%)$ e dentre aqueles que relataram algum tipo de deficiência (26= $25,24 \%)$, metade (13=50\%) declararam possuir deficiência auditiva. Quanto às condições de saúde, verifica-se que a maioria declarou possuir algum problema (85= 82,52\%); faz uso de algum tipo de medicação $(88=85,44 \%)$, realizou consulta médica no último ano (97=94,17\%) e 58 idosos (56,31\%) realizaram consulta odontológica no último ano.

Tabela 1: Distribuição das variáveis sociodemográfica dos idosos participantes de um grupo de convivência no Municipio de Belém-PA (2017)

\begin{tabular}{|c|c|c|c|}
\hline Variável & $N$ & $\%$ & P-Valor(1) \\
\hline \multicolumn{4}{|l|}{ Sexo } \\
\hline Feminino & 93 & 90,29 & \multirow{2}{*}{$<0.0001^{* *}$} \\
\hline Masculino & 10 & 9,71 & \\
\hline \multicolumn{4}{|l|}{ Faixa Etária } \\
\hline $60-65$ & 24 & 23,30 & \multirow{5}{*}{$0.0141^{*}$} \\
\hline $65-70$ & 13 & 12,62 & \\
\hline $70-75$ & 32 & 31,07 & \\
\hline $75-80$ & 21 & 20,39 & \\
\hline 80 ou mais & 13 & 12,62 & \\
\hline \multicolumn{4}{|c|}{ Sabe ler e escrever? } \\
\hline Sim & 103 & 100,00 & \multirow{2}{*}{ - } \\
\hline Não & 0 & 0,00 & \\
\hline \multicolumn{4}{|c|}{ Escolaridade (anos de estudo) } \\
\hline $1 \mathrm{~A} 3$ anos & 4 & 3,88 & \multirow{3}{*}{$<0.0001^{\star \star}$} \\
\hline $4 \mathrm{~A} 8$ anos & 21 & 20,39 & \\
\hline 8 anos ou mais & 78 & 75,73 & \\
\hline \multicolumn{4}{|l|}{ Cor/Raça } \\
\hline Pardo & 48 & 46,60 & \multirow{6}{*}{$<0.0001^{\star \star}$} \\
\hline Branco & 34 & 33,01 & \\
\hline Negro & 16 & 15,53 & \\
\hline Amarelo & 2 & 1,94 & \\
\hline Indigena & 2 & 1,94 & \\
\hline $\begin{array}{l}\text { Não Declarado } \\
\text { (ND) }\end{array}$ & 1 & 0,97 & \\
\hline
\end{tabular}




\begin{tabular}{|c|c|c|c|}
\hline \multicolumn{4}{|c|}{ Situação Conjugal } \\
\hline Viúvo (a) & 33 & 32,04 & \multirow{6}{*}{$<0.0001^{* *}$} \\
\hline $\begin{array}{l}\text { Casado (a) / } \\
\text { convivio c/ } \\
\text { parceiro (a) }\end{array}$ & 30 & 29,13 & \\
\hline Solteiro (a) & 24 & 23,30 & \\
\hline Divorciado (a) & 11 & 10,68 & \\
\hline Outra & 4 & 3,88 & \\
\hline Separado (a) & 1 & 0,97 & \\
\hline \multicolumn{4}{|c|}{ Tem alguma deficiência? } \\
\hline Sim & 26 & 25,24 & \multirow{2}{*}{$<0.0001^{\star \star}$} \\
\hline Não & 77 & 74,76 & \\
\hline \multicolumn{4}{|l|}{ Qual? } \\
\hline Auditiva & 13 & 50,00 & \multirow{3}{*}{$0.0190^{\star}$} \\
\hline Visual & 11 & 42,31 & \\
\hline Física & 2 & 7,69 & \\
\hline \multicolumn{4}{|c|}{ Mora com quem? } \\
\hline Com parentes & 65 & 63,11 & \multirow{3}{*}{$<0.0001^{* \star}$} \\
\hline $\begin{array}{l}\text { Cônjuge ou } \\
\text { companheiro }\end{array}$ & 10 & 9,71 & \\
\hline Sozinho (a) & 28 & 27,18 & \\
\hline \multicolumn{4}{|c|}{ Aposentado ou pensionista? } \\
\hline Sim & 89 & 86,41 & \multirow{2}{*}{$<0.0001^{* *}$} \\
\hline Não & 14 & 13,59 & \\
\hline \multicolumn{4}{|l|}{ Renda Mensal } \\
\hline $\begin{array}{l}\text { Menos de um } \\
\text { salário }\end{array}$ & 4 & 3,88 & \multirow{4}{*}{$<0.0001^{* *}$} \\
\hline Um salário & 25 & 24,27 & \\
\hline Dois salários & 19 & 18,45 & \\
\hline $\begin{array}{l}\text { Três ou mais } \\
\text { salários }\end{array}$ & 55 & 53,40 & \\
\hline \multicolumn{4}{|c|}{ Tem algum problema de saúde? } \\
\hline Sim & 85 & 82,52 & \multirow{2}{*}{$<0.0001^{* *}$} \\
\hline Não & 18 & 17,48 & \\
\hline \multicolumn{4}{|c|}{ Utiliza algum tipo de medicação? } \\
\hline Sim & 88 & 85,44 & \multirow{2}{*}{$<0.0001^{* \star}$} \\
\hline Não & 15 & 14,56 & \\
\hline
\end{tabular}

Realizou consulta médica no último ano?

\begin{tabular}{|c|c|c|c|}
\hline Sim & 97 & 94,17 & \multirow{2}{*}{$<0.0001^{\star \star}$} \\
\hline Não & 6 & 5,83 & \\
\hline \multicolumn{4}{|c|}{ Realizou consulta odontológica no último ano? } \\
\hline Sim & 58 & 56,31 & \multirow{2}{*}{$0.2370 \mathrm{~ns}$} \\
\hline Não & 45 & 43,69 & \\
\hline
\end{tabular}

(1)Teste Qui-quadrado de Pearson para tendência ( $p$-valor $<0.05)$.

** Valores Altamente significativos; *Valores Significativos; NS Valores Não Significativos.

$\mathrm{Hl}$ : Existe tendência significativa entre as frequências $(p<0.05)$.

Na Tabela 2 está a distribuição dos escores de qualidade de vida (OV) dos domínios do WHOQOL-BREF dos idosos participantes da pesquisa, avaliados por meio do instrumento.
As duas primeiras questões do Whoqol-bref, apesar de fazerem parte do escore global, estratificam a população de idosos quanto às suas percepções em relação à qualidade de vida e condição de saúde.

Considerando que o escore médio é avaliado da seguinte forma: a qualidade de vida necessita melhorar (quando for 1 até 2,9); é regular (3 até 3,9); é boa (4 até 4,9) e é muito boa (5). Em relação aos domínios da qualidade de vida, obteve maior escore para o domínio percepção da qualidade de vida (POV) $\operatorname{com}(\mu=4.15 \pm 0.61)$ e relações sociais (RS) $\operatorname{com}(\mu=4.05 \pm$ $0.46)$, e menor escore em meio ambiente (MA) com ( $\mu=3.73$ $\pm 0.47)$.

Tabela 2: Nivel da qualidade de vida dos idosos participantes de um grupo de convivência no Município de Belém-PA (2017), segundo os domínios qualidade de vida, saúde, físico, social, psicológico e ambiental mensurados pelo questionário de Whoqol-Bref.

\begin{tabular}{lll} 
Dominio & Média & DP \\
\hline POV & 4.15 & 0.61 \\
\hline SS & 3.85 & 0.75 \\
\hline DF & 3.93 & 0.54 \\
\hline DP & 3.93 & 0.50 \\
\hline RS & 4.05 & 0.46 \\
\hline MA & 3.73 & 0.47 \\
\hline
\end{tabular}

POV - Percepção da qualidade de vida; SS - Satisfação com a saúde; DF - Domínio Físico; DP - Domínio Psicológico; RS Relação Social; MA - Meio Ambiente.

\section{DISCUSSÃO}

Esse estudo apresentou características similares da predominância do sexo feminino de outros estudos que afirmam que este fato está relacionado com o processo de feminização da velhice, onde pode ser justificado pela maior preocupação das mulheres com o autocuidado e pela maior procura pelo serviço de saúde. Além disso os homens estão mais expostos a situações de violência, agressões e acidentes e acabam não chegando a faixas etárias longevas na mesma proporção que as mulheres ${ }^{(10,11)}$.

Quanto ao resultado encontrado em escolaridade e renda mensal, pode estar ligado ao fato de que para participação nesse centro de convivência é necessário dispor de uma taxa. Dessa forma, os idosos com mais escolaridade e maior poder aquisitivo têm mais facilidade em adentrar ao grupo.

Em relação ao convívio familiar dos idosos, como foi visto, a maioria reside com familiares e isso representa um elemento importante para a saúde e bem-estar da pessoa idosa, é na família muitas vezes que o idoso encontra o apoio necessário para as dificuldades encontradas no dia-a-dia, fazendo com 
que estes se sintam acolhidos e mais seguros.

O grande número de viúvas frequentando o grupo de convivência pode estar relacionado na tentativa de evitar o isolamento, a dor da perda, a solidão, por isso os grupos sociais para estes idosos não são apenas momentos de lazer, ao contrário, é de suma importância possibilitando-lhes ressocialização.

Durante o processo do envelhecimento podem ocorrer algumas perdas sensoriais, como as dificuldades para ouvir frequências mais altas, resultantes da degeneração das células dos órgãos dos sentidos. Essa disfunção auditiva denominada presbiacusia, pode ser observada nos idosos entrevistados, pois os mesmos tiveram dificuldade de compreensão, esse fato implica em efeitos negativos, visto que prejudica a comunicação, afetando consequentemente as relações sociais e a qualidade de vida ${ }^{(12)}$.

Além da presbiacusia os idosos alegaram ser acometidos por algumas doenças crônicas tais como: Hipertensão Arterial Sistêmica; Diabete Mellitus; Osteoporose e Doenças Cardiovasculares, tornando-os dependentes do uso contínuo de medicamentos e de acompanhamento médico. As doenças crônicas não-transmissiveis (DCNT) podem acometer a funcionalidade do idoso, comprometendo a capacidade de realização das atividades básicas de vida diária (ABVD), tais como tarefas de autocuidado; tomar banho, vestir-se, alimentar-se e também o comprometimento das atividades instrumentais de vida diária (AIVDs), como, por exemplo, fazer compras, telefonar, utilizar meios de transporte, realizar tarefas domésticas, preparar uma refeição, cuidar do próprio dinheiro. A perda dessa capacidade funcional que pode ser ocasionada por essas doenças crônicas, que quando não controladas levam a perda da autonomia e independência interferindo na qualidade vida desses idosos ${ }^{(13)}$.

O fato desses idosos terem algum tipo de doença crônica não interferiu na percepção de qualidade de vida dos mesmos, visto que o domínio qualidade de vida obteve maior escore. Pode-se dizer que este resultado está relacionado com a auto percepção dos idosos deste estudo, pois relatam que sua qualidade de vida é boa, devido eles se considerarem ativos, satisfeitos com a saúde, terem o controle das doenças que thes acometem, felizes e acolhidos pelos familiares.

Em relação aos domínios relações sociais e meio ambiente, resultados parecidos foram encontrados em uma pesquisa com idosos residentes em Palmas- PR, onde também obtiveram escores maiores no domínio RS $(\mu=3,88 \pm 0,57)$ e menor no MA $(\mu=3,37 \pm 0,48)(14)$. O menor valor em MA deve sugerir a dificuldade de adaptação no meio onde estes residem, fazendo necessário o planejamento do meio urbano de acordo com a Norma Brasileira (NBR) Regulamentadora 9050 da Associação Brasileira de Normas Técnicas (ABNT), com intuito de permitir ambientes saudáveis e seguros para essa população, ou seja, criar espaços adaptados, como ambientes mais iluminados, sinalizados, com rampas de acesso, corrimão, pisos antiderrapantes, de modo a evitar quedas, acidentes e consequentemente problemas de saúde.Com o processo do envelhecimento as atividades executadas no dia-a-dia acabam se tornando mais difíceis de serem realizadas, logo, ter um ambiente favorável tornase indispensável para manter a autonomia, independência e consequentemente um envelhecimento com qualidade ${ }^{(15)}$.

O resultado da pesquisa em relação ao escore no domínio RS é possível que esteja ligado com as maiores oportunidades que os idosos pesquisados têm de participar das atividades disponibilizadas no centro de convivência, a relação diária entre eles e os seus familiares, as amizades desenvolvidas na comunidade e nos centros de convivência, proporcionando aos idosos uma sensação de bem-estar, contribuindo com o processo de envelhecimento e com a saúde emocional ${ }^{(16)}$.

Desse modo, a qualidade de vida é um constructo subjetivo que possui várias dimensões, é a percepção, compreensão e satisfação que o indivíduo considera ter em relação a vida. Comumente a qualidade de vida está associada à autoestima, bem-estar pessoal, podendo influenciar no estado de saúde, no modo de vida, nas relações sociais e familiares, no nível socioeconômico e na satisfação que estes têm com a vida ${ }^{(17)}$.

\section{Limitações do estudo}

Como limitação pode-se apontar o número de pessoas estudadas e o fato da pesquisa ter sido com idosos com uma situação econômica boa, o que facilita os mesmos participarem deste Centro de Convivência, uma vez que é necessário dispor de uma taxa mensal para usufruir do espaço, o que impossibilita a generalização dos dados. Entretanto a pesquisa servirá como estímulo para mais pesquisas nessa área e para criação de mais espaços como esse a fim de tornarem-se acessiveis a pessoas que não tenham condições financeiras.

\section{Contribuições do estudo para a prática}

Sabe-se que a população idosa está em contínuo crescimento, sendo assim é necessário que o governo, profissionais e a sociedade priorizem esse acontecimento afim de aprimorar os serviços socioeconômico e de saúde já existentes, planejar e ofertar cada vez mais serviços com equidade a população que thes proporcione um envelhecimento com qualidade de vida. Essa pesquisa irá contribuir para o maior conhecimento e incentivo a profissionais da área da saúde e gestores a continuarem estimulando os idosos e pessoas em geral a participarem de centros de convivências e outros setores da sociedade, 
engajando-se cada vez mais na comunidade e incorporando cada vez mais hábitos saudáveis com o intuito de alcançar um envelhecimento ativo.

\section{CONCLUSÃO}

No presente estudo verificou-se através do instrumento Whoqol-bref que os idosos participantes do grupo de convivência têm uma boa qualidade de vida, onde os domínios PQV e relações sociais foram avaliados positivamente com maior escore e como menor escore foi o meio ambiente.

EsseCentro de Convivência oferecepormeio das atividades propostas, mais possibilidades de interações sociais entre os idosos, trocas de experiências, lazer, promove saúde, estimula hábitos saudáveis tornando-os mais ativos e dispostos a aceitar o processo de senescência, proporcionando-lhes acima de tudo bem-estar físico e psicológico.

O estudo feito nesse centro mostrou a importância da implementação de mais grupos que atendam todas as classes sociais e que tenham boa acessibilidade, visto que nessa pesquisa o escore meio ambiente foi baixo, esse fato poder ter ocorrido não somente pelos relatos de que o ambiente onde esses idosos vivem é barulhento, de difícil acesso, sujo, mas também pelo fato desse espaço não dispor de uma melhor acessibilidade, como rampas, elevadores, corrimão.

No entanto, notou-se a ausência do profissional de enfermagem, levando a reflexão da importância do mesmo nos grupos de convivência, pois estes contribuem com a melhoria da qualidade de vida dos idosos através de ações de cuidados, aconselhamento, prevenção de doenças e agravos.

Diante do exposto, conclui-se que a participação desses idosos nesse centro de convivência os motiva de alguma forma a não perderem a autonomia, a capacidade de desenvolver as atividades de vida diária e possibilita o resgate do convívio social, refletindo na melhoria da qualidade de vida.

\section{Contribuição dos autores}

Concepção e desenho, análise e interpretação dos dados, redação do artigo, revisão crítica, revisão final: Marcela Alves de Oliveira, Hemellen Ferreira Ribeiro, Nadia Pinheiro da Costa.

\section{REFERÊNCIAS}

1. Mendes JLV, Silva SC, Silva GR, Santos NAR. O aumento da população idosa no Brasil e o envelhecimento nas últimas décadas: Uma revisão da literatura. REV. EDUC. MEIO AMB. SAÚ. [Internet]. 2018 [cited JAN/ MAR]:8(1):13-26. Available from: www.faculdadedofuturo.edu.br/revistal/ index.php/remas/article/view/165

2. Cruz CB, Macedo KCS, Medeiros MP. Lucas GKS. Ativa idade. Revista Brasileira da Educação Profissional e Tecnológica [Internet]. 2018 [cited 2018 Mar];1;1-10. Availabe from: http://www2.ifrn.edu.br/ojs/index.php/ RBEPT/article/download/6876/pdf

3. Cantele AB, Araújo BN, Pellizzari G, Bidel RMR. Envelhecimento ativo: o conhecimento dos técnicos de enfermagem da estratégia saúde da família. Revista Saúde e Desenvolvimento [Internet]. 2017 [cited 2017];11(9): 156171. Availabe from: https://www.uninter.com/revistasaude/index.php/ saudeDesenvolvimento/article/download/784/467

4. Carvalho AMB, Cardosos JA, Silva FAA, Lira JAC, Carvalho SM. Qualidade de vida no trabalho da equipe de enfermagem do centro cirúrgico. Enferm Foco [Internet]. 2018 [cited 2018 Mai 10]:9(3):35-41. Available from: http:// revista.cofen.gov.br/index.php/enfermagem/article/download/1159/458

5. Schoffen LP. Santos WL. A importância dos grupos de convivência para os idosos como instrumento para manutenção da saúde. Rev. Cient. Sena Aires [Internet]. 2018 [cited 2018 Out-Dez]:7(3):160-171. Availabe from: http://revistafacesa.senaaires.com.br/index.php/revisa/article/ view/317/227

6. Fontelles MJ, Simões MG, Almeida JC, Fontelles RGS. Metodologia da pesquisa: diretrizes para o cálculo do tamanho da amostra. Revista Paraense de Medicina [Internet].2010 [cited 2010 Abr]:24(2):57-64. Availabe from: http://files.bvs.br/upload/S/0101-5907/2010/v24n2/ a2125.pdf

7. Chaves RN, Lima PV, Valença TDC, Santana ES, Marinho MS, Reis LA. Perda cognitiva e dependência funcional em idosos longevos residentes em instituições de longa permanência. C ogitare Enferm. [Internet]. 2017 [cited $2017 \mathrm{Jan} / \mathrm{mar}$ ]; 22(1): 01-09. Available from: https://revistas.ufpr.br/ cogitare/article/view/48497/pdf

8. Cavalcante DG, Oliveira DV, Antunes MD, Prati ARC. Análise da qualidade de vida em idosas praticantes de dança. Revista Interdisciplinar de Promoção da Saúde [Internet]. 2018 [cited 2018 Jan /Mar.]; 1(1): 23-31. Available from: https://online.unisc.br/seer/index.php/ripsunisc/article/ view/11944
9. Rodrigues CFS, Lima FJC, Barbosa FT. Importância do uso adequado da estatística básica nas pesquisas clínicas. Rev Bras Anestesiol.[Internet]. 2017 [cited 2017 Abr 10];67(6):619-625. Available from: http://www.scielo. br/pdf/rba/v67n6/pt_0034-7094-rba-67-06-0619.pdf

10. Junior AGS, Pedro JO, Oliveira MC, Furlan CR, Nascimento FG. Basseler TC, et.al. Ca-racterização sociodemográfica e a autopercepção das condições de saúde de idosos. Rev enferm UFPE [Internet]. 2018 [cited 2018 Mar]; 12(3):692-700. Available from: https://periodicos.ufpe. br/revistas/revistaenfermagem/article/download/230161/28026

11. Barbosa RL, Silva TDCS, Santos MF, Carvalho FR, Marques RVDA, Matos Junior EM. Perfil sociodemográfico e clínico dos idosos de um Centro de Convivência. Revista Kairós-Gerontologia [Internet]. 2018 [cited 2018]; 21(2):357-373. Available from: https://revistas.pucsp.br/kairos/article/ download/40968/27640

12. Guarisco LPC. Dalpubel D. Labanca L, Chagas MHN. Percepção da perda auditiva: utili-zação da escala subjetiva de faces para triagem auditiva em idosos. Ciência \& Saúde Coletiva [Internet] 2017 [cited 2017 Dez 10]; 22(11):3579-3588. Available from: https://www.scielosp.org/pdf/ csc/2017.v22n1l/3579-3588/pt

13. Gavasso WC, Beltrame V. Capacidade funcional e morbidades referidas: uma análise comparativa em idosos. Rev. Bras. Geriatr. Gerontol. [Internet] 2017 [cited 2017 Mai 19]; 20(3): 399-409. Available from: http:// www.scielo.br/pdf/rbgg/v20n3/pt_1809-9823-rbgg-20-03-00398.pdf 14. Ribeiro CG, Ferreti F, Sá CA. Qualidade de vida em função do nivel de atividade física em idosos urbanos e rurais. Rev. Bras. Geriatr. Gerontol. [Internet]. 2017 [cited 2017 Abr 24]; 20(3): 330-339. Available from: http:// www.scielo.br/pdf/rbgg/v20n3/pt_1809-9823-rbgg-20-03-00330.pdf 15. Pagliuca LMF, Lima BS, Silva JM, Cavalcante LM, Martins MC, Araújo TL. Acesso de idosos às unidades de atenção primária à saúde. REME - Rev Min Enferm.[Internet]. 2017 [cited 2017];21:1-5. Available from: http://www. reme.org.br/artigo/detalhes/1157

16. Silva RL, Santos CC, Prazeres LR. Dança Sênior: uma alternativa para melhorar a quali-dade de vida dos idosos. Revista Kinesis [Internet]. 2018 [cited 2018 Agost 21];36(2);73-82. Available from: https://periodicos.ufsm. br/kinesis/article/view/30596

17. Fidalgo AR, Landim LFS, Melo DM. Suporte social e qualidade de vida no envelhecimen-to: uma revisão sistemática. Rev. Estação Cientifica [Internet]. 2018 [cited $2018 \mathrm{Jan} / J u n$ ]; 19;1-19.Available from: http://portal. estacio.br/media/3730426/suporte-social-e-qualidade-de-vida-noenvelhecimento.pdf 